\title{
Synchronous gastric and duodenal metastases from head and neck squamous cell carcinoma: a unique presentation of upper gastrointestinal bleeding
}

\author{
Nicholas P. Tarangelo a, C. Andrew Kistlerb, Zachary Daitch', Wei Jiang ${ }^{d}$, Daniel M. Quirk ${ }^{\text {b }}$ \\ Thomas Jefferson University Hospital; Temple University Hospital, Philadelphia, PA, USA
}

\begin{abstract}
Metastatic disease to the stomach or duodenum is an infrequent diagnosis, and head and neck squamous cell carcinoma (HNSCC) is one of the least common primary malignancies that lead to gastric or duodenal metastases. We report the case of a 65-year-old man with human immunodeficiency virus infection and previously diagnosed HNSCC who presented with melena. The patient had a percutaneous endoscopic gastrostomy tube placed 3 months prior to his presentation. Laboratory testing was significant for normocytic anemia and a digital rectal examination was positive for melena. Esophagogastroduodenoscopy revealed numerous cratered nodules with contact bleeding in the stomach as well as the duodenum that appeared malignant. Biopsies of the gastric and duodenal nodules were positive for $\mathrm{p} 40$ and CK 5/6, consistent with metastatic squamous cell carcinoma.
\end{abstract}

Keywords Duodenum, gastrointestinal bleeding, human immunodeficiency virus, squamous cell carcinoma, stomach

Ann Gastroenterol 2018; 31 (1): 1-3

\section{Introduction}

Metastatic disease to the stomach or duodenum is an infrequent diagnosis, with prior autopsy studies estimating the incidence of gastric metastases to be $0.2-0.7 \%$ [1]. The most common cancers that metastasize to the stomach are melanoma, breast, esophageal, and lung [2]. Malignancy is an uncommon cause of upper gastrointestinal (GI) bleeding (UGIB). Gastric and duodenal malignancies have been estimated to cause only $1.9-4.5 \%$ of all cases of UGIB $[3,4]$.

Head and neck squamous cell carcinoma (HNSCC) most commonly metastasizes to the lung, bone, and liver [5]. It is

Departments of ${ }^{a}$ Medicine, Thomas Jefferson University Hospital (Nicholas P. Tarangelo); ${ }^{\mathrm{b}}$ Medicine, Division of Gastroenterology and Hepatology, Thomas Jefferson University Hospital (C. Andrew Kistler, Daniel M. Quirk); ${ }^{\mathrm{C}}$ Medicine, Temple University Hospital (Zachary

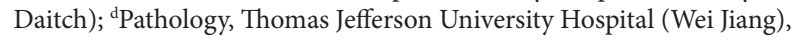
Philadelphia, PA, USA

Conflict of Interest: None

Patient Consent: The patient consented to having his case presented for publication.

Correspondence to: Nicholas P. Tarangelo, 833 Chestnut St, Suite 701, Philadelphia, PA, 19107, USA, e-mail: nicholas.tarangelo@gmail.com

Received 11 December 2017; accepted 5 January 2018; published online 8 February 2018

DOI: https://doi.org/10.20524/aog.2018.0235 one of the least common primary malignancies that lead to gastric or duodenal metastases. We report a rare case of UGIB secondary to metastatic HNSCC to the stomach and duodenum in a patient with human immunodeficiency virus (HIV) infection.

\section{Case report}

A 65-year-old man with HIV (CD4 count 354 cells $/ \mathrm{mm}^{3}$ ) on antiretroviral therapy and squamous cell carcinoma of the right tonsil, tongue, and pharynx presented to the emergency department after having several melenic bowel movements. He was found to be hemodynamically stable and to have benign findings on the abdominal examination. Gastric lavage through his percutaneous endoscopic gastrostomy (PEG) tube returned heme-positive coffee ground material. Digital rectal examination was positive for melena. Laboratory data were significant for normocytic anemia of $6.8 \mathrm{~g} / \mathrm{dL}$, blood urea nitrogen of $24 \mathrm{mg} / \mathrm{dL}$ and creatinine of $0.6 \mathrm{mg} / \mathrm{dL}$. The platelet count, prothrombin time, and partial thromboplastin time were within normal limits.

The patient was diagnosed with HNSCC six months prior to admission and had undergone two months of chemotherapy and radiation therapy followed by tracheostomy and PEG tube placement. Transoral robotic surgery and bilateral neck dissection were performed one month prior to admission. He was found to have metastases to local lymph nodes at that time. 
Esophagogastroduodenoscopy revealed numerous cratered nodules with contact bleeding in the stomach (Fig. 1) as well as in the duodenum (Fig. 2,3) that appeared malignant. These nodules were diffusely present in the stomach and ranged in size from $0.5-2.0 \mathrm{~cm}$ in diameter. There were no ulcerations or nodules underneath the PEG tube site. No active bleeding was observed and no endoscopic therapy was performed. Biopsies of both gastric and duodenal nodules were diffusely positive for p40 and CK 5/6 (Fig. 4), consistent with metastatic squamous cell carcinoma. The patient was transfused one unit of packed red blood cells and was eventually discharged to home hospice.

\section{Discussion}

The incidence of gastric metastases varies in the literature. Prior autopsy studies from the 1960s and 1990s found the incidence of gastric metastases from solid organ malignancies to range from $0.2-0.7 \%$ [1]. However, a more recent study from 2001 found the rate of gastric metastases to be $5.4 \%$ among patients diagnosed at autopsy [2]. It is unclear whether this variability in the incidence of gastric metastases represents a true change in the metastatic tropism of solid organ malignancies or rather is a result of more effective treatments

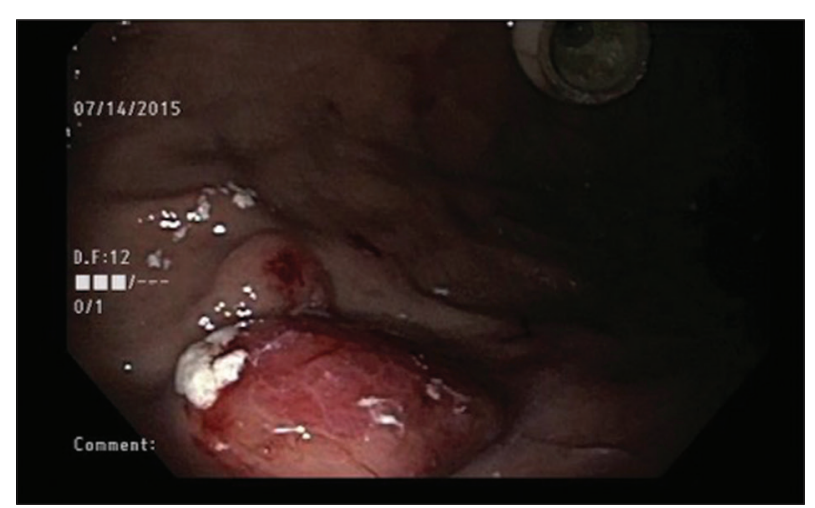

Figure 1 Cratered nodule in the stomach with contact bleeding (bottom left) and percutaneous endoscopic gastrostomy internal bumper, internal site (top right)

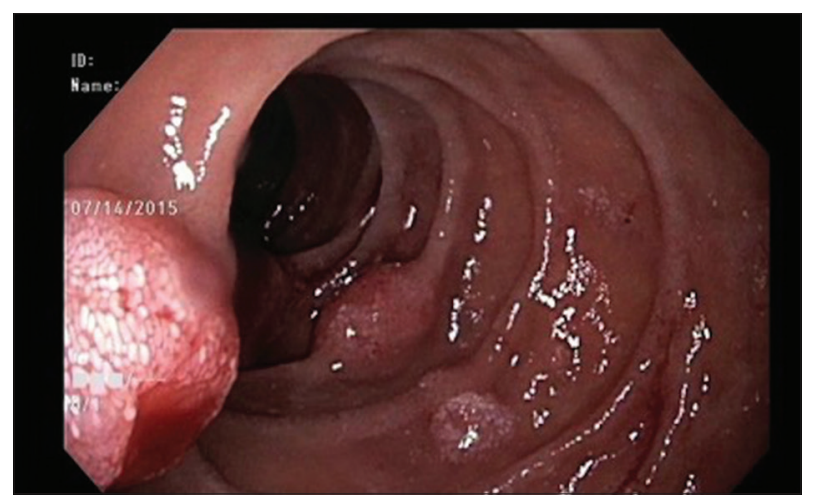

Figure 2 Cratered nodules with contact bleeding in the second part of the duodenum leading to improved mortality. In the case of most solid organ malignancies, advances in oncologic treatment have improved mortality from the time of diagnosis, thus allowing more opportunity for the development of metastatic disease. This change in the natural history of many solid organ malignancies may account for the rising rate of gastric metastases in more recent studies [2].

As uncommon as gastric metastases from HNSCC are, duodenal metastases are even less common. A 2010 review of all reported cases of HNSCC metastases to the small bowel yielded a total of 12 cases [6]. Of these cases, only one showed metastasis to the duodenum. Of the remaining cases of HNSCC metastases to the small bowel, seven were metastatic to the ileum, three were metastatic to the jejunum, and one case did not specify the site of the small bowel metastasis. The rarity of HNSCC metastases to the duodenum highlights the unique nature of our case.

The link between HIV and certain malignancies has been well documented in the literature, with certain malignancies, such as Kaposi sarcoma and squamous cell carcinoma of the cervix, being established as HIV-defining illnesses [7]. Prior studies have examined the characteristics of HNSCC in

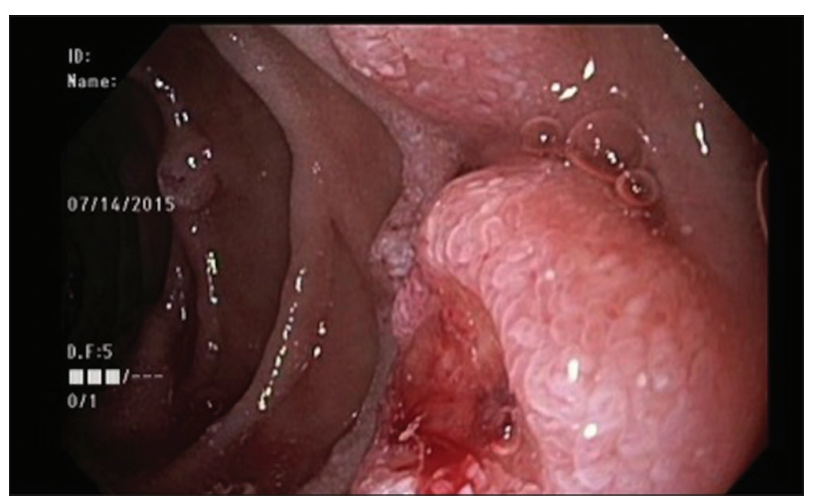

Figure 3 Additional cratered nodules in the second part of the duodenum

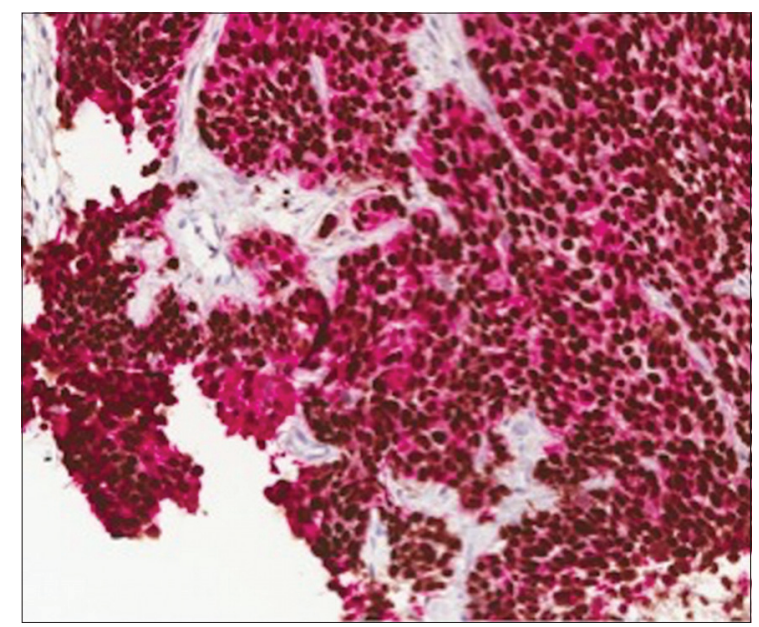

Figure 4 Duodenal biopsy showing squamous cell carcinoma with staining positive for $\mathrm{p} 40$ (brown nuclear stain) and CK5/6 (red membranous stain) 
patients with HIV infection. Patients with HNSCC and HIV were found to have an earlier mean age of onset, more advanced disease at the time of presentation, and worse mortality at one and two years compared to patients without concurrent HIV infection [8]. There is a paucity of data regarding the pattern of metastatic disease in patients with HNSCC and HIV. However, it can be postulated that defects in local immune surveillance in patients with HIV may result in an increased likelihood of distant metastases, including metastases to the GI tract.

The introduction of foreign devices, specifically PEG tubes, has been suggested as a risk factor and likely mechanism for seeding metastases in distant sites $[9,10]$. Prior studies suggest that direct placement is superior to pull-through placement for PEG tubes in patients with HNSCC, having a lower risk of seeding distant sites [9]. Our patient did not have metastases at the stoma site, as seen in the majority of patients with seeding due to PEG tube placement; however, in the setting of this rare distribution of metastases the possibility of seeding the upper GI tract from PEG placement should be considered.

Several aspects of this case are unique. Metastatic disease as a cause of UGIB is uncommon. PEG placement as a possible mechanism for distal seeding of the GI tract has been documented in the literature, but the anatomic distribution of metastases described in our case is uncommon. This case is likely the only documented case of HNSCC with metastases both to the stomach and the duodenum. The rarity of this pattern of metastatic disease in HNSCC raises the question of whether immunocompromise in the setting of HIV infection increases the likelihood of distant metastasis; however, further studies are needed to evaluate this possibility.

\section{References}

1. Green LK. Hematogenous metastases to the stomach. A review of 67 cases. Cancer 1990;65:1596-1600.

2. Oda, Kondo H, Yamao T, et al. Metastatic tumors to the stomach: analysis of 54 patients diagnosed at endoscopy and 347 autopsy cases. Endoscopy 2001;33:507-510.

3. Savides TJ, Jensen DM, Cohen J, et al. Severe upper gastrointestinal tumor bleeding: endoscopic findings, treatment, and outcome. Endoscopy 1996;28:244-248.

4. Loftus EV, Alexander GL, Ahlquist DA, Balm RK. Endoscopic treatment of major bleeding from advanced gastroduodenal malignant lesions. Mayo Clin Proc 1994;69:736-740.

5. Ferlito A, Shaha AR, Silver CE, Rinaldo A, Mondin V. Incidence and sites of distant metastases from head and neck cancer. ORL J Otorhinolaryngol Relat Spec 2001;63:202-207.

6. Dwivedi RC, Kazi R, Agrawal N, et al. Comprehensive review of small bowel metastasis from head and neck squamous cell carcinoma. Oral Oncol 2010;46:330-335.

7. Safai B, Diaz B, Schwartz J. Malignant neoplasms associated with human immunodeficiency virus infection. CA Cancer J Clin 1992;42:74-95.

8. Singh B, Balwally AN, Shaha AR, Rosenfeld RM, Har-El G, Lucente FE. Upper aerodigestive tract squamous cell carcinoma. The human immunodeficiency virus connection. Arch Otolaryngol Head Neck Surg 1996;122:639-643.

9. Cappell MS. Risk factors and risk reduction of malignant seeding of the percutaneous endoscopic gastrostomy track from pharyngoesophageal malignancy: a review of all 44 known reported cases. Am J Gastroenterol 2007;102:1307-1311.

10. Ellrichmann M, Sergeev P, Bethge J, et al. Prospective evaluation of malignant cell seeding after percutaneous endoscopic gastrostomy in patients with oropharyngeal/esophageal cancers. Endoscopy 2013;45:526-531. 